\title{
Diretrizes para o Desenvolvimento de Serious Games: Um Mapeamento Sistemático da Literatura
}

\author{
Wendell Soares Pereira', Gilberto Amado de Azevedo Cysneiros Filho', Yuska Paola \\ Costa Aguiar ${ }^{2}$
}
${ }^{1}$ Programa de Pós-Graduação em Informática Aplicada (PPGIA) - Universidade Federal Rural de Pernambuco (UFRPE) - Recife - Pernambuco - Brasil
${ }^{2}$ Departamento de Ciências Exatas (DCX) - Universidade Federal da Paraíba (UFPB) Rio Tinto - Paraíba - Brasil
\{Wendell.pereira, yuska\}@dcx.ufpb.br, g.cysneiros@gmail.com

\begin{abstract}
Serious games are increasingly being used as facilitators in educational environments, going beyond the entertainment and fun. However, maintaining the playfulness of a serious game is a challenge towards the established learning objectives, and it is relevant to emphasize aspects related to gameplay and learning. This article presents 53 guidelines to assist in the development of serious games. These were identified in a systematic mapping of the literature that included 19 studies among the 194 obtained from the search bases.
\end{abstract}

Resumo. É crescente a utilização de serious games como facilitadores em ambientes educacionais, perpassando o entretenimento e a diversão. Porém, manter a ludicidade de um serious game é um desafio frente aos seus objetivos de aprendizagem estabelecidos, sendo relevante enfatizar aspectos relacionados à jogabilidade e à aprendizagem. Para tanto, neste artigo são apresentadas 53 diretrizes para auxiliar no desenvolvimento de serious games. Estas foram identificadas a partir de um mapeamento sistemático da literatura que contemplou 19 estudos entre os 194 retornos obtidos nas bases de busca.

\section{Introdução}

Jogos são conceituados por Neves e Alves (2015) como atividades cuja natureza ou finalidade visam fins recreativos, diversão e entretenimento, em qualquer contexto ou ambiente. Com o crescimento do uso de recursos digitais em diversos segmentos da sociedade, os jogos tiveram seu conceito ampliado de acordo em adequação à realidade atual. Os jogos digitais (digital games) mantêm os princípios dos jogos tradicionais, no entanto suas ações e regras são controladas por um programa de computador [Lucchese e Ribeiro, 2009].

Dentre os diferentes tipos de jogos, encontram-se os jogos sérios (serious games $S G s$ ). Estes têm como objetivo central a aprendizagem, independente do contexto de aplicação (educação, treinamento, saúde, etc.), não sendo o propósito primário apenas a diversão [Chen e Michael, 2005; Whyte et al., 2015; Dörner et al., 2016]. Nesse sentido, os SGs buscam aliar os objetivos educacionais e as características dos jogos para promover a generalização do aprendizado e criar ambientes agradáveis e imersivos. Sendo comum o emprego dos princípios de design de videogames [Whyte et al., 2015; Freitas, 2006].

No Brasil, em 2017 foram identificados pelo II Censo da Indústria Brasileira de Jogos Digitais (2018) 964 jogos digitais desenvolvidos, representando um aumento de 28\% considerando a produção do ano anterior. Entre estes, aproximadamente 50\% (463) foram 
VIII Congresso Brasileiro de Informática na Educação (CBIE 2019)

Anais do XXX Simpósio Brasileiro de Informática na Educação (SBIE 2019)

categorizados como serious games [Sakuda e Fortim, 2018]. No entanto, jogos educacionais, ainda que projetados por empresas especialistas em games, quando não consideram aspectos pedagógicos e de aprendizagem, são atraentes e divertidos, mas falham quanto aos objetivos de aprendizagem [Van Eck, 2006]. Encontrar a sinergia entre a diversão e a pedagogia nos jogos educacionais têm se mostrado uma tarefa difícil [Savi e Ulbricht, 2008]. Por esse motivo, faz-se necessário conhecer os elementos que caracterizam os serious games para que o desenvolvimento destes alcancem o equilíbrio entre aprendizado e diversão, sendo efetivo ao seu propósito.

Nesse sentido, a fim de conhecer as boas práticas para o desenvolvimento de serious games disponíveis na literatura científica, um mapeamento sistemático de literatura foi conduzido. Um corpus de 19 artigos foi construído para a identificação de diretrizes direcionadas ao design e desenvolvimento de serious games. Um total de 135 boas práticas foram extraídas do corpus, analisadas, agrupadas por similaridade e classificadas segundo os princípios de usabilidade clássicos [Nielsen, 1995; Bastien e Scapin, 1998]. O resultado consiste em um conjunto de 53 diretrizes enquanto uma ferramenta útil para guiar a tomada de decisão de designers e desenvolvedores de serious games.

Este artigo está organizado em quatro seções, incluindo esta. Na seção 2, são expostos os processos metodológicos do mapeamento sistemático. Na seção 3, encontram-se os resultados e discussões, incluindo a lista de diretrizes propostas classificados sob a ótica dos princípios de usabilidade. Por fim, na seção 4, estão as considerações finais, contemplando as limitações deste trabalho e suas possibilidades de continuação.

\section{Revisão Sistemática da Literatura}

Uma revisão sistemática da literatura (RLS) é um meio para identificar, avaliar e interpretar a pesquisa disponível relevante para determinadas questões de pesquisa, área temática ou fenômeno de interesse [Kitchenham e Charters, 2007]. O protocolo de pesquisa segundo Biolchini et al. (2005) contempla: (i) especificação das questões de pesquisa; (ii) as bases de dados selecionadas; (iii) critérios de inclusão e exclusão dos estudos de interesse; (iv) especificação dos dados a serem extraídos e analisados.

Diante do objetivo de identificar as boas práticas de design e desenvolvimento de serious games, as questões de pesquisa que nortearam a pesquisa foram: (QP1): Com qual objetivo os estudos foram realizados (prover diretrizes com base na literatura, diretrizes a partir de um estudo de caso ou experimento, diretrizes a partir do desenvolvimento de algum serious game, ou ainda, desenvolver algum serious game com base em diretrizes)? (QP2): Quais foram as boas práticas para serious games citadas nos estudos analisados? E (QP3) Para qual contexto estas boas práticas devem ser aplicadas (ambiente de aplicação do serious game, usuário ou área específica do conhecimento)?

Para a busca, foi aplicada uma string ${ }^{1}$ em cinco bases de dados, a saber: (i) Sience Direct; (ii) ACM Digital Library; (iii) IEEE Xplore Digital Library; (iv) PubMed; (v) Compendex (Engineering Village). Foram selecionados os artigos cujos títulos contivessem o termo "serious games" ou "serious game", e cujo abstract ou palavras-chaves apresentassem os termos "design recommendations", "guidelines", "heuristic", "best

\footnotetext{
${ }^{1}$ String utilizada para as buscas nas bases de dados: ("serious games" OR "serious game") AND ("design recommendations" OR guidelines OR heuristic OR "best practices" OR "key principles")
} 
VIII Congresso Brasileiro de Informática na Educação (CBIE 2019)

Anais do XXX Simpósio Brasileiro de Informática na Educação (SBIE 2019)

practices" ou "key principles". A string sofreu alterações sintáticas, porém não semânticas, para que esta se adaptasse às normas de cada base de dados.

Para direcionar a seleção de estudos de forma a responder às questões de pesquisa, foram estabelecidos critérios de inclusão (CI) e de exclusão (CE) a serem aplicados na filtragem dos estudos de interesse. Um artigo foi incluído quando não infringiu algum dos critérios de exclusão e respeitava ao menos um dos critérios de inclusão. Os critérios utilizados foram: (CI1) o artigo é escrito em inglês, espanhol, francês ou português; (CI2) o artigo descreve o processo de concepção e/ou desenvolvimento do serious game; (CE1) o artigo é duplicado (ou versão menos detalhada/mais antiga de outro artigo); (CE2) o artigo não é completo (menor de 6 páginas); (CE3) o artigo é indisponível de forma gratuita; (CE4) o artigo não aborda serious games; $e$, (CE5) o artigo não apresenta boas práticas para o design e desenvolvimento de serious games.

As informações extraídas dos estudos selecionados foram: (i) objetivo do estudo realizado; (ii) identificação das boas práticas descritas para a concepção do SG ou nas lições aprendidas a partir desse desenvolvimento; (iii) contexto de aplicação das boas práticas; (iv) ano de publicação; e (v) referência completa do artigo.

\section{Resultados e Discussão}

A busca nas bases de dados resultou em 194 estudos, sendo 131 proveniente da Compendex, 26 da Science Direct, 22 da IEEE, 13 da PubMed e 2 da ACM. Após realização da filtragem com aplicação dos critérios de inclusão e exclusão definidos no protocolo de pesquisa, o corpus da RSL foi definido contemplando 19 artigos (Tabela 1) - aproveitamento de 9.79\% sobre o retorno obtido com aplicação inicial da string de busca.

Tabela 1 - Lista dos trabalhos selecionados.

\section{ID. Referência do Artigo}

A01: Tomé, R. M., Pereira, J. M., \& Oliveira, M. (2014, October). Using serious games for cognitive disabilities. In International Conference on Serious Games Development and Applications(pp. 34-47). Springer, Cham.

A02: Van Broeckhoven, F., Vlieghe, J., \& De Troyer, O. (2015, September). Mapping between pedagogical design strategies and serious game narratives. In 2015 7th International Conference on Games and Virtual Worlds for Serious Applications (VS-Games) (pp. 1-8). IEEE.

A03: Tong, T., Chignell, M., \& Sieminowski, T. (2015). Case study: a serious game for neurorehabilitation assessment. Procedia Computer Science, 69, 125-131.

A04: Georgieva, G., Arnab, S., Romero, M., \& de Freitas, S. (2015). Transposing freemium business model from casual games to serious games. Entertainment Computing, 9, 29-41.

A05: Tsekleves, E., Cosmas, J., \& Aggoun, A. (2016). Benefits, barriers and guideline recommendations for the implementation of serious games in education for stakeholders and policymakers. British Journal of Educational Technology, 47(1), 164-183.

A06: Cano, S., Arteaga, J. M., Collazos, C. A., Gonzalez, C. S., \& Zapata, S. (2016). Toward a methodology for serious games design for children with auditory impairments. IEEE Latin America Transactions, 14(5), 2511-2521.

A07: Ushaw, G., Eyre, J., \& Morgan, G. (2017, April). A paradigm for the development of serious games for health as benefit delivery systems. In 2017 IEEE 5th International Conference on Serious Games and Applications for Health (SeGAH) (pp. 1-8). IEEE.

A08: Lameras, P., Arnab, S., Dunwell, I., Stewart, C., Clarke, S., \& Petridis, P. (2017). Essential features of serious games design in higher education: Linking learning attributes to game mechanics. British journal of educational technology, 48(4), 972-994. 
VIII Congresso Brasileiro de Informática na Educação (CBIE 2019)

Anais do XXX Simpósio Brasileiro de Informática na Educação (SBIE 2019)

A09: Wilson, A. S., Broadbent, C., McGrath, B., \& Prescott, J. (2017). Factors Associated with Player Satisfaction and Educational Value of Serious Games. In Serious Games and Edutainment Applications (pp. 513-535). Springer, Cham.

A10: Ravyse, W. S., Blignaut, A. S., Leendertz, V., \& Woolner, A. (2017). Success factors for serious games to enhance learning: a systematic review. Virtual Reality, 21(1), 31-58.

A11: Abidin, S. R. Z., Noor, S. F. M., \& Ashaari, N. S. (2017, November). Guidelines of brainbased learning through serious game for slow reader students. In 2017 6th International Conference on Electrical Engineering and Informatics (ICEEI) (pp. 1-6). IEEE.

A12: Tavakoli, S., Alghassi, A., Perinpanayagam, S., \& Garcia, D. A. (2017, April). Skill development in the wind energy sector: A serious game development approach. In 2017 IEEE Global Engineering Education Conference (EDUCON) (pp. 2-9). IEEE.

A13: Machado, M. D. C., Ferreira, R. L. R., \& Ishitani, L. (2018). Heuristics and Recommendations for the Design of Mobile Serious Games for Older Adults. International Journal of Computer Games Technology, 2018.

A14: Malliarakis, C., Tomos, F., Shabalina, O., \& Mozelius, P. (2018, October). Andragogy and EMOTION: 7 key factors of successful serious games. In ECGBL 2018 12th European Conference on Game-Based Learning (p. 371). Academic Conferences and publishing limited.

A15: Gouin-Vallerand, C., Ferreira, S. M., \& Hotte, R. (2018, November). Towards a mobile serious game environment for children self-learning. In Proceedings of the 4th EAI International Conference on Smart Objects and Technologies for Social Good(pp. 100-105). ACM.

A16: Tsikinas, S., \& Xinogalos, S. (2018, April). Designing effective serious games for people with intellectual disabilities. In 2018 IEEE Global Engineering Education Conference (EDUCON) (pp. 1896-1903). IEEE.

A17: Fanfarelli, J. R., McDaniel, R., \& Crossley, C. (2018). Adapting UX to the design of healthcare games and applications. Entertainment Computing, 28, 21-31.

A18: Savazzi, F., Isernia, S., Jonsdottir, J., Di Tella, S., Pazzi, S., \& Baglio, F. (2018). Design and implementation of a Serious Game on neurorehabilitation: Data on modifications of functionalities along implementation releases. Data in brief, 20, 864-869.

A19: Robertson, J., Macvean, A., Fawkner, S., Baker, G., \& Jepson, R. G. (2018). Savouring our mistakes: Learning from the FitQuest project. International journal of child-computer interaction, 16, 55-67.

\subsection{Quanto ao objetivo dos estudos selecionados}

Durante o processo de extração de dados os estudos foram classificados segundo seu objetivo central. Desta forma (i) 8 artigos descreveram boas práticas com base na literatura; (ii) 6 artigos indicam boas práticas com base em experimento/estudo de caso; (iii) 2 artigos propuseram boas práticas com base no desenvolvimento de um SG; e (iv) 3 artigos aplicaram boas práticas disponíveis na literatura para o desenvolvimento de um SG. Este cenário nos leva a inferir que existe uma lacuna no tocante à proposição de boas práticas provenientes da prática de desenvolvimento de SG. Além disso, considerando o baixo número de artigos que executam boas práticas (disponíveis na literatura) em projetos práticos de desenvolvimento de SG, nos faz questionar sobre a validação das proposições existentes.

\subsection{Diretrizes para o desenvolvimento de Serious Games}

Na extração dos dados foram identificadas 135 boas práticas para o design e desenvolvimento de SG. Cada uma destas foi associada a um ID único com referência ao seu artigo de origem (IDArtigo.IDguideline, por exemplo: A04.01 faz referência à primeira boa prática descrita no artigo 04.). 
VIII Congresso Brasileiro de Informática na Educação (CBIE 2019)

Anais do XXX Simpósio Brasileiro de Informática na Educação (SBIE 2019)

\subsubsection{Agrupamento de diretrizes por similaridade}

Seguida à identificação das boas práticas, estas foram analisadas e agrupadas por semelhança. Ou seja, quando a mesma boa prática foi extraída de artigos diferentes, estas foram agrupadas, dando origem a uma única diretriz. Este processo teve como propósito eliminar duplicidade nas proposições, reduzindo a quantidade de diretrizes sem impactar na limitação da cobertura das mesmas. Das 53 diretrizes propostas para o design e desenvolvimento de serious games, 22 são resultantes de boas práticas citadas em pelo menos dois artigos do corpus analisado. As 31 restantes possuem única ocorrência no corpus. Na Tabela 2 a informação disponível consiste em: <ID_Guideline: Título da diretriz; número de ocorrências nos artigos analisados; [lista dos IDs das boas práticas que foram agrupadas para dar origem à diretriz em questão]>

\section{Tabela 2 - Diretrizes para o desenvolvimento de Serious Games.}

\begin{tabular}{|c|}
\hline Diretrizes para Design e Desenvolvimento de Serious Games \\
\hline G01: Regras e objetivos claros, desde o início do jogo; 4; [A04.01; A09.05; A13.01; A17.06] \\
\hline $\begin{array}{l}\text { G02: Maestria rápida sobre o jogo, fácil de aprender; 6; [A04.02; A09.01; A11.01; A13.11; } \\
\text { A14.03; A18.03] }\end{array}$ \\
\hline G03: Adaptabilidade de acordo com as ações do jogador; 4; [A04.03; A06.05; A07.01; A10.03] \\
\hline G04: Contextualização com os temas cotidianos; 3; [A01.03; A04.04; A18.06] \\
\hline $\begin{array}{l}\text { G05: Prover feedback ao usuário; 9; [A01.05; A04.07; A06.04; A07.04; A08.01; A09.10; } \\
\text { A10.05; A11.09; A18.10] }\end{array}$ \\
\hline $\begin{array}{l}\text { G06: Escolha de tecnologia acessível para o público alvo (tablet, smartphone, computador); } 2 \text {; } \\
\text { [A03.01; A07.02] }\end{array}$ \\
\hline G07: Informações visíveis sobre o progresso do jogador; 3; [A03.02; A08.05; A13.05] \\
\hline $\begin{array}{l}\text { G08: Fazer uso do game-level, partindo do mais simples ao mais complexo; 6; [A03.04; } \\
\text { A05.03; A08.02; A11.06; A13.09; A16.04] }\end{array}$ \\
\hline $\begin{array}{l}\text { G09: Correspondência entre os objetivos do jogo e os objetivos de aprendizagem; 3; [A05.01; } \\
\text { A06.01; A14.06] }\end{array}$ \\
\hline $\begin{array}{l}\text { G10: Controle do usuário sobre alguns aspectos do jogo; } 5 \text { [A01.02;A06.02; A09.06; A13.12; } \\
\text { A18.12] }\end{array}$ \\
\hline G11: Recompensas; 5; [A01.05; A06.07; A08.03; A12.04; A13.06] \\
\hline $\begin{array}{l}\text { G12: Prover diferentes modelos de linguagem comunicativa (geralmente verbal ou visual); } 2 \text {; } \\
\text { [A06.09; } A 18.15]\end{array}$ \\
\hline $\begin{array}{l}\text { G13: Contexto do jogo de fácil compreensão - StoryLine, personagens, cenário, avatar, etc.; } 4 \\
\text { [A07.05; } A 10.01 ; A 11.02 ; A 14.04]\end{array}$ \\
\hline G14: Armazenamento de dados para análise posterior; 2; [A07.06; A16.05] \\
\hline $\begin{array}{l}\text { G15: Variedade nos desafios apresentados para evitar que o jogo se torne enfadonho, } \\
\text { investindo também na diversão; } 5 ;[A 09.02 ; A 11.08 ; A 12.02 ; A 13.10 ; A 14.02]\end{array}$ \\
\hline $\begin{array}{l}\text { G16: Prover imersão através de áudios e imagens realistas de alta qualidade; } 4 ;[A 10.02 ; \\
A 11.05 ; A 13.04 ; A 16.02]\end{array}$ \\
\hline $\begin{array}{l}\text { G17: Learning Outcomes - Exibir, desde o início, o que o jogador tem aprendido a cada etapa } \\
\text { do jogo; } 3 ;[\text { [A04.05; A09.09; A12.07] }\end{array}$ \\
\hline G18: Interatividade no jogo - Promover o aprendizado colaborativo; 2; [A10.04; A11.10] \\
\hline
\end{tabular}


VIII Congresso Brasileiro de Informática na Educação (CBIE 2019)

Anais do XXX Simpósio Brasileiro de Informática na Educação (SBIE 2019)

G20: Pré-requisitos devem ser minimizados, mas, se necessários, devem ser ensinados; 2; [A01.06; A13.02]

G21: Instruções de uso devem ser fornecidas, um tutorial por exemplo; 3; [A13.03; A16.06; A18.04]

G22: Envolver o usuário final no processo de criação, geralmente, testando o jogo e indicando possíveis modificações; 2; [A17.05; A19.01]

G23: Avaliação de necessidades;1; [A02.01]

G24: Preparação de matriz de mudanças de objetivo;1; [A02.02]

G25: Seleção de teoria para métodos e estratégias;1; [A02.03]

G26: Desenvolvimento do programa de intervenção;1; [A02.04]

G27: Planejamento para a adoção, implementação e sustentabilidade;1; [A02.05]

G28: Deve-se haver vários tipos de avaliação ou pequenos jogos (suíte de jogos) para acomodar uma maior variedade de usuários;1; [A03.03]

G29: Elementos surpresa;1; [A06.06]

G30: Avaliação por Ranking entre os usuários;1; [A06.08]

G31: Escolher um gênero de jogo [puzzle, platform, role playing];1; [A07.07]

G32: Especificar dispositivo de entrada;1; [A07.03]

G33: Elaborar questões e respostas especificando a área de conhecimento;1; [A12.01]

G34: Score como learning outcome; 1 [A12.03]

G35: Tempo limite para as tarefas; $1 ;$ [A12.05]

G36: Cores específicas para cada nível;1; [A12.06]

G37: Evitar penalizações por erros;1; [A13.07]

G38: Desafios de acordo com a idade dos usuários;1; [A13.08]

G39: A jogabilidade precisa ser longa para garantir o interesse do usuário;1; [A13.13]

G40: Os primeiros 10 minutos de jogo devem ser óbvios, promovendo bons resultados imediatos; 1 ; [A13.14]

G41: Fluxo natural entre o aprendizado e a diversão;1; [A14.07]

G42: Manter a motivação e o engajamento;1; [A14.08]

G43: Definir a estrutura das atividades; 1 ; $A$ 14.09]

G44: Definir um modelo pedagógico a ser aplicado;1; [A14.10]

G45: Considerar testes de usabilidade estruturados com frameworks robustos; 1 ; $A 17.01]$

G46: Determinar como os modelos formativos e somativos podem ajudar no estudo do engajamento do usuário final; $1 ;$ [A17.02]

G47: Conduzir análises de Front-end para determinar ações do usuário final e instrutores;1; [A17.03]

G48: Utilização do design iterativo para manter o ritmo de evolução do jogo;1; [A17.04]

G49: Consistência entre cores, fontes e imagens;1; [A18.01]

G50: Evitar que o usuário precise memorizar grande quantidade de informação;1; [A18.09]

G51: Prevenção de erro - Exibir mensagens informando o erro e como ele pode ser resolvido; $1 ;$ [A18.13] 
VIII Congresso Brasileiro de Informática na Educação (CBIE 2019)

Anais do XXX Simpósio Brasileiro de Informática na Educação (SBIE 2019)

G52: Produção de um protótipo;1; [A19.02]

G53: Uma vez construída uma ferramenta mais sólida, uma avaliação mais duradoura deve ser feita; $1 ;$ [A19.03]

$\mathrm{Na}$ tentativa de entender as características que levaram algumas diretrizes (G22...G53) a não serem consideradas por mais estudos, foi realizada uma classificação onde as diretrizes poderiam se enquadrar em uma das 3 categorias: (a) específica para SGs; (b) para softwares em geral, que são intrínsecas ao processo de concepção; ou (c) específica para um domínio de aplicação, por exemplo para o ensino de matemática, artes, etc. A maioria (14/31) das diretrizes com ocorrência única no corpus são oriundos de boas práticas de engenharia de software, portanto implícito ao desenvolvimento de qualquer tipo de software. Houveram ainda 11/31 diretrizes que eram específicas para serious games, enquanto 6/31 diretrizes apresentaram características específicas para o domínio de aplicação.

\subsection{Quanto ao contexto de aplicação dos diretrizes}

Considerando que as diretrizes resultantes desta pesquisa foram extraídas de boas práticas relacionadas a serious games para diversos contextos de aplicação (saúde, educação, treinamento, etc.) e público alvo distinto (crianças e adultos, com ou sem deficiência), se fez necessário analisar quão específicas estas são para o cenário à qual pertencem. Desta forma, é possível identificar a generalidade ou especificidade do mesmo, auxiliando a decisão de manutenção ou não destas na proposição deste trabalho de pesquisa. Para o público alvo, têm-se uma variedade de perfis contemplados, a saber: pessoas com deficiência intelectual (1); crianças com deficiência auditiva (1); crianças com deficiência intelectual (1); profissionais do setor de energia eólica (1); idosos (1); e crianças (2). Para a área de conhecimento, têm-se: saúde (4); educação (4); treinamento tecnológico. Além destes, temse um Quiz e um Exergames. Quatro artigos do corpus analisado não dispõe de informações específicas sobre o público alvo e área de conhecimento.

\subsection{Classificação dos diretrizes}

De forma a facilitar a curva de aprendizado de projetistas e desenvolvedores de software que se lançam no desafio de concepção de serious games, foi realizado um mapeamento entre as diretrizes propostas para SG e os princípios de usabilidade amplamente utilizados e consolidados na área de Interação Humano-Computador (IHC). O mapeamento considerou as heurísticas de Nielsen (1995) e os critérios ergonômicos de Bastien e Scapin (1993).

$\mathrm{Na}$ Figura 2 tem-se a ilustração resultante do mapeamento realizado entre as diretrizes (extremidades laterais) as heurísticas de Nielsen (centro à esquerda) e os critérios ergonômicos de Bastien e Scapin (centro à direita). No total, 13 diretrizes puderam ser mapeadas, entre estes, os que tiveram correspondência com os dois autores (7/13) foram identificados com uma cor, enquanto os demais (6/13) permaneceram marcados em preto. As diretrizes que tiveram correspondência com os dois autores considerados foram identificados também pela ligação entre os critérios marcados com a mesma cor. 
VIII Congresso Brasileiro de Informática na Educação (CBIE 2019)

Anais do XXX Simpósio Brasileiro de Informática na Educação (SBIE 2019)

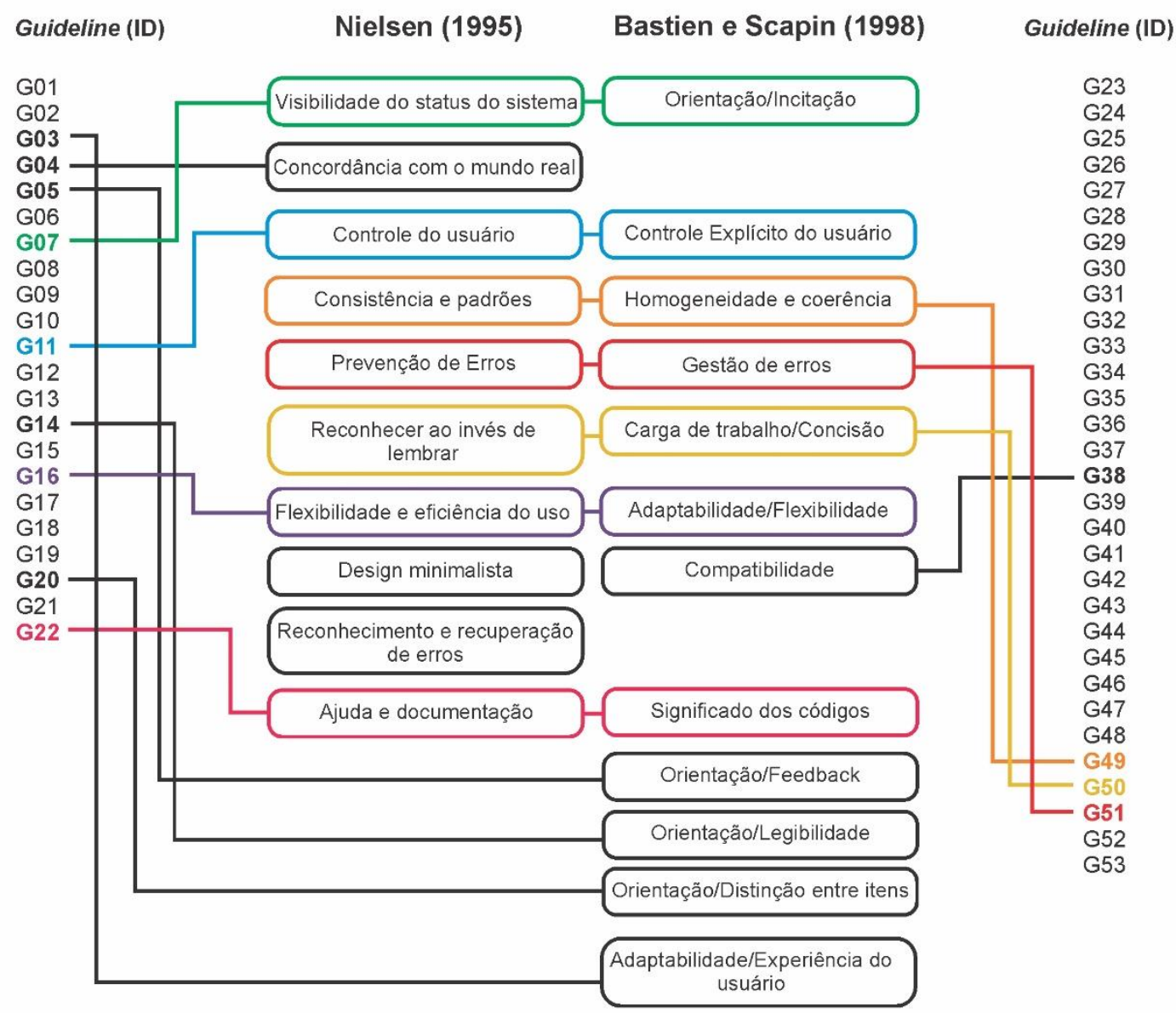

Figura 2. Mapeamento das diretrizes para design e desenvolvimento de serious games.

\section{Considerações Finais}

Este trabalho contempla a descrição e os resultados de um mapeamento sistemático de literatura para identificação de diretrizes para o design e desenvolvimento de serious games. De forma adicional, foi realizado um mapeamento entre as diretrizes encontradas com os princípios de usabilidade consagrados na área de IHC, as heurísticas de Nielsen (1995) e os critérios ergonômicos de Bastien e Scapin (1993). A proposição das diretrizes e o respectivo mapeamento foram motivados pela importância de prover aos projetistas de serious games um instrumental que guie os mesmos e que potencialize o sucesso dos produtos gerados diante da desafiadora tarefa de conciliar e equilibrar aprendizado e diversão dos serious games, fazendo com que os mesmos sejam efetivos ao seu propósito.

O mapeamento sistemático de literatura partiu de um total de 194 estudos selecionados nas principais bases de busca científica da área de Computação, mas não exclusivo à estas. Um corpus composto por 19 estudos que foram selecionados e analisados, por respeitarem os critérios de inclusão e exclusão estabelecidos em protocolo, cujo conteúdo se apresentava suficiente para responder às questões de pesquisa definidas. A extração de dados resultou em 135 boas práticas associadas à serious games, que foram analisadas e agrupadas por similaridade em 53 diretrizes. Estes, foram mapeados segundo os princípios de usabilidade. 
Apesar de obtida a lista de diretrizes, esta limita-se a características extraídas da literatura, o que pode contrastar com a realidade de desenvolvimento de serious games encontradas nas empresas que os desenvolvem. Para isto, como continuidade deste estudo, propõe-se um survey junto às empresas, startups e laboratórios que concebem serious games. Este trabalho limitou-se igualmente a apresentar as diretrizes sem uma validação prévia, o que pode ser contemplado em um futuro desenvolvimento de um serious game considerando a aplicação destas diretrizes.

\section{Referências}

Bastien, J.M.C and Scapin, D.L. (1993). Critères Ergonomiques pour l'Évaluation d'Interfaces Utilisateurs (version 2.1). Technical report Ndeg.156, May 1993. INRIA. Programme 3 Artificial intelligence, cognitive systems, and man-machine interaction

Biolchini, J.; Mian, P. G., Natali, A. C. C. and Travassos, G. H. (2005) Systematic Review in Software Engineering. Technical Report, RT - ES679/05. Systems Engineering and Computer Science Department. COPPE/UFRJ.

Chen, S., and Michael, D. (2005). Proof of learning: Assessment in serious games. Retrieved October, 17, 2008.

Dörner, R.; Göbel, S.; Effelsberg, W.; Wiemeyer, J. (Eds.). (2016). Serious games: foundations, concepts and practice. Springer.

Freitas, Sara I. (2006). Using games and simulations for supporting learning. Learning, media and technology, vol. 31, no 4, p. 343-358.

Kitchenham, B.; Charters, S. (2007). Guidelines for performing Systematic Literature Reviews in Software Engineering. Technical Report EBSE-2007-01. Disponível em: <encurtador.com.br/dKOP4> Acesso em 28/08/2019.

Lucchese, F. and Ribeiro, B. (2009). Conceituação de jogos digitais. São Paulo. Disponível em: < http://www.dca.fee.unicamp.br/ martino/disciplinas/ia369/trabalhos/t1g3.pdf $>$. Acesso em 28/08/2019

Neves, I. B.; Alves, L. (2015) Exergames e tratamento da obesidade: uma análise sobre a plataforma Tango H. Anais do Seminário Tecnologias Aplicadas a Educação e Saúde, v. 1, n. 1 .

Nielsen, J. (1995). 10 usability heuristics for user interface design. Nielsen Norman Group, $1(1)$.

Sakuda, L. O. and Fortim, Ivelise. (2018). II censo da indústria brasileira de jogos digitais. Ministério da cultura e Homo-Ludens: São Paulo. Disponível em: $<$ https://nuvem.cultura.gov.br/index.php/s/mdxtGP2QSYO7VMz\#pdfviewer/> Acesso em 14/05/2019.

Savi, R. and Ulbricht, V. R. (2008). Jogos digitais educacionais: benefícios e desafios. Renote, 6(1).

Van Eck, R. (2006). Digital game-based learning: It's not just the digital natives who are restless. EDUCAUSE review, 41(2), 16.

Whyte, Elisabeth M.; Smyth, Joshua M.; et Scherf, K. Suzanne. (2015). Designing serious game interventions for individuals with autism. Journal of autism and developmental disorders, vol. 45 , no 12 , p. $3820-3831$. 